\title{
First results with the IOTA3 imaging interferometer: The spectroscopic binaries $\lambda$ Vir and WR 140
}

\author{
J. D. Monnier ${ }^{1}$, W. A. Traub ${ }^{2}$, F. P. Schloerb ${ }^{3}$, R. Millan-Gabet ${ }^{4}$, J.-P. Berger ${ }^{5}$, \\ E. Pedretti ${ }^{2}$, N. P. Carleton ${ }^{2}$, S. Kraus ${ }^{3}$, M. G. Lacasse ${ }^{2}$, M. Brewer ${ }^{3}$, S. Ragland ${ }^{2}$, \\ A. Ahearn ${ }^{2}$, C. Coldwell ${ }^{2}$, P. Haguenauer ${ }^{6}$, P. Kern ${ }^{5}$, P. Labeye ${ }^{7}$, L. Lagny ${ }^{5}$, F. Malbet ${ }^{5}$, \\ D. Malin ${ }^{3}$, P. Maymounkov ${ }^{2}$, S. Morel ${ }^{8}$, C. Papaliolios ${ }^{2}$, K. Perraut ${ }^{5}$, M. Pearlman ${ }^{2}$, \\ I. L. Porro ${ }^{9}$, I. Schanen ${ }^{10}$, K. Souccar ${ }^{3}$, G. Torres ${ }^{2}$, and G. Wallace ${ }^{3}$
}

\begin{abstract}
We report the first spatially-resolved observations of the spectroscopic binaries $\lambda$ Vir and WR 140, which includes the debut of aperture-synthesis imaging with the upgraded three-telescope IOTA interferometer. Using IONIC-3, a new integrated optics beam combiner capable of precise closure phase measurement, short observations were sufficient to extract the angular separation and orientation of each binary system and the component brightness ratio. Most notably, the underlying binary in the prototypical colliding-wind source WR 140 (WC7 $+\mathrm{O} 4 / 5)$ was found to have a separation of $\sim 13$ milli-arcseconds with a position angle consistent with the images of the 2001 dust shell ejection only if the WolfRayet star is fainter than the $\mathrm{O}$ star at $1.65 \mu \mathrm{m}$. We also highlight $\lambda$ Vir whose peculiar stellar properties of the Am star components will permit direct testing of current theories of tidal evolution when the full orbit is determined.
\end{abstract}

\footnotetext{
${ }^{1}$ monnier@umich.edu: University of Michigan (Astronomy), 500 Church St, Ann Arbor, MI 48109-1090

${ }^{2}$ Harvard-Smithsonian Center for Astrophysics, 60 Garden St, Cambridge, MA, 02138, USA

${ }^{3}$ University of Massachusetts, Amherst

${ }^{4}$ Michelson Science Center, California Institute of Technology, Pasadena, CA

${ }^{5}$ Laboratoire d'Astrophysique de Grenoble, 414 Rue de la Piscine 38400 Saint Martin d'Heres, France

${ }^{6}$ Alcatel Space Industries, Cannes, France

${ }^{7}$ LETI-CEA, Grenoble, France

${ }^{8}$ European Southern Observatory, Germany

${ }^{9}$ Massachusetts Institute of Technology, Cambridge, MA

${ }^{10}$ IMEP-INPG, Grenoble, France
} 
Subject headings: instrumentation: interferometers - techniques: interferometric

- binaries: spectroscopic - stars: individual (WR 140, $\lambda$ Vir),

\section{Introduction}

Only two optical (visible and infrared) interferometers have published results combining three or more separated telescopes, the Cambridge Optical Aperture Synthesis Telescope (Baldwin et al. 1996) and the Navy Prototype Optical Interferometer (Benson et al. 1997). Combining more than two telescopes is crucial in order to measure the closure phase, a phase quantity that is uncorrupted by atmospheric turbulence and necessary for image reconstruction in "very long baseline interferometry" (Jennison 1958; Monnier 2000); indeed, closure phase lies at the heart of so-called "self-calibration" techniques widely used in the radio (e.g., Readhead et al. 1980; Cornwell \& Wilkinson 1981).

Here we present the first scientific results of the upgraded 3-telescope IOTA interferometer (Traub et al. 2003), with the new integrated-optics combiner IONIC-3 (Berger et al. 2003) for precise measurements of visibilities and closure phases. IOTA has much greater near-infrared sensitivity (demonstrated $\mathrm{H}$ mag 7) than the other imaging arrays, allowing new observational programs including imaging of young stellar objects, mass determinations of low-mass binaries, and mapping photospheric surface structures of red giants. After describing our novel instrumentation, we present the first resolved observations for the A-star binary $\lambda$ Vir and colliding wind system WR 140 and discuss the significance of our findings. Here we alert the astronomical community to the need for radial velocity follow-up of these specific sources as well as to the new research opportunities available with the IOTA3 array.

\section{Observations and Analysis}

All the data presented were obtained using the IOTA (Infrared-Optical Telescope Array) interferometer (Traub et al. 2003), which is operated by a consortium of institutions, most notably the Smithsonian Astrophysical Observatory and the University of Massachusetts at Amherst. The 0.45-m telescopes are movable among 17 stations along 2 orthogonal linear arms (telescopes A \& C can move along the 35-m northeastern arm, while Telescope B moves along the $15-\mathrm{m}$ southeastern arm), allowing an aperture $35 \mathrm{~m} \times 15 \mathrm{~m}$ to be synthesized (corresponding to an angular resolution of $\sim 5 \times 12$ milliarcseconds at $1.65 \mu \mathrm{m}$ ). Capping a long development (e.g., Schloerb 1990), the third telescope ("C") was fully incorporated on 
UT 2002 February 23 and now allows visibilities on three baselines and one closure phase to be measured simultaneously. The initial observations used a prototype integrated optical (IO) combiner (e.g., Kern et al. 1997; Berger et al. 2001) which operated only with polarized light. The upgraded combiner IONIC-3 corrected this deficiency in 2002 November, and all observations presented here were obtained in unpolarized light.

We only just introduce IONIC-3 (Berger et al. 2003) here; an engineering paper with detailed description of the $\mathrm{IO}$ component and its performance is in preparation (Berger et al. 2004). Light from each telescope is focused into a dedicated single-mode fiber, and the three fibers are aligned using a silicon v-groove array mated to the planar waveguides on the IO device. The optical circuit acts to split the light from each telescope before recombining each telescope pair (AB,BC,AC) at 3 IO couplers. This "pair-wise" combination scheme leads to 6-interferometric channels (two for each baseline), where the interference fringes are temporally-modulated by scanning piezo mirrors placed in two of the telescope beams; a sensitive HgCdTe (Rockwell PICNIC) array (Pedretti et al. 2004a, submitted) detects the signals, and data are recorded by a vme-based control system linked to a Sun workstation.

We followed established observing procedures, as outlined in previous IOTA publications (Coudé du Foresto et al. 1997; Millan-Gabet et al. 2001): observations of target and nearby calibrator stars were interspersed to calibrate slowly varying system visibilities and instrumental closure phases. The science targets presented here were observed using a standard $\mathrm{H}$ band filter $\left(\lambda_{0}=1.65 \mu \mathrm{m}, \Delta \lambda=0.30 \mu \mathrm{m}\right)$; Table 1 contains other observing details. Figure 1 shows the $(\mathrm{u}, \mathrm{v})$-coverage obtained during each epoch for each science target.

Reduction of the $\mathcal{V}^{2}$ data was carried out using an Interactive Data Language (IDL) implementation of the method outlined by Coudé du Foresto et al. (1997). In short, we measured the power spectrum of each interferogram (proportional to the target $\mathcal{V}^{2}$ ), after correcting for intensity fluctuations and subtracting out bias terms from read noise, residual intensity fluctuations, and photon noise (e.g., Perrin 2003). To assure good closure phase measurement, we required that interferograms be detected on $\geq 2$ baselines, a condition nearly always maintained by a realtime fringe packet "tracker" (Pedretti et al. 2004b, in preparation). Lastly, the data pipeline applied a correction for the variable flux ratios for each baseline by using a transfer matrix (based on single-beam flux measurements; e.g., Coudé du Foresto et al. 1997; Monnier 2001). We have studied the absolute calibration accuracy by observing single stars of known size and found a systematic error of $\Delta \mathcal{V}^{2}=0.10$ (e.g., $5 \%$ error in visibility for unresolved sources) under the worst observing conditions; this error has been combined (in quadrature) with the statistical error for fitting purposes. Improved analysis methods should achieve $\lesssim 1 \%$ errors eventually, since we are using single-mode fibers.

We followed the method of Baldwin et al. (1996) for calculating the complex triple 
amplitude in deriving the closure phase, explicitly guaranteeing "fringe frequency closure" $\left(\nu_{A B}+\nu_{B C}+\nu_{C A}=0\right)$. While photon-noise bias correction is not necessary for the IONIC-3 ("pair-wise") combiner, the instrumental closure phase must be determined empirically. The miniature dimensions of the IO component have minimized drifts to less than 1 degree over many hours; chromaticity effects, however, limit our absolute precision when the calibrator and source are not of the same spectral type due to different "effective wavelengths." Engineering tests indicate that the instrumental closure phase $\left(\Phi_{C P}\right)$ varies systematically by $1.4 \pm 0.3$ degrees between a hot star (B8) and a cool star (M3) when using the broadband $\mathrm{H}$ filter. While we minimize these errors by using calibrators matched to the spectral type of the target, a conservative systematic error of $\Delta \Phi_{C P}=0.75$ degs was adopted here to approximate our expected residual level of miscalibration. Lastly, the sign of our closure phase $\left(\Phi_{A B}+\Phi_{B C}+\Phi_{C A}\right.$ for baseline triangle connecting telescopes $\left.\mathrm{A} \rightarrow \mathrm{B} \rightarrow \mathrm{C} \rightarrow \mathrm{A}\right)$ was calibrated using the well-known binary stars Capella and Matar (Hummel et al. 1994, 1998).

\section{Results and Discussion}

With calibrated $\mathcal{V}^{2}$ and closure phases for each target, a static binary star model (separation, position angle, brightness ratio) was fitted to the data for each given epoch, assuming the calibration errors given in the previous section and ignoring bandwidth-smearing effects. Table 2 contains the results of our fits based on a maximum-likelihood approach giving equal weights to the visibility data and closure phases. Explicitly, the probability of a given set

of parameters was approximated by: Probability $\propto \exp \left(-\frac{\chi_{\mathrm{Vis}}^{2}}{\operatorname{minimum}\left(\chi_{\mathrm{Vis}^{2}}^{2}\right)}-\frac{\chi_{\mathrm{CP}}^{2}}{\operatorname{minimum}\left(\chi_{\mathrm{CP}}^{2}\right)}\right)$. This normalization is equivalent to increasing the data errors to force the $\chi^{2} / \mathrm{DOF}=1$, and conservatively estimates errors in the context of an incomplete error model. The most probable values with 1-sigma errors bars (68.4\% confidence interval) were calculated for each parameter (separation, position angle, brightness ratio) by integrating over the other variables, a standard practice referred to as marginalizing over these variables.

Based on their brightness and color temperatures, the component stars of both binaries are expected to be unresolved here $(\lesssim 0.5$ mas $)$; this assumption, as well as neglecting bandwidth-smearing, may not remain valid if the data precision is improved significantly. The $\mathcal{V}^{2}$ and closure phases for a subset of the data are shown in Figure 2 along with the best-fitting model prediction. We note that $\lambda$ Vir, which has a $\sim 207$ day period, is not completely static during each observing "epoch." With complete orbital phase coverage, this deficiency will be corrected by solving for the full orbital elements by fitting directly to the visibilities, closure phases, and radial velocities, a procedure outlined in detail by Hummel et al. (1998) and Boden et al. (1999). 
The radio community developed interferometric imaging techniques (e.g., hybrid mapping) capable of reobtaining lost individual phases (e.g., Haniff et al. 1987). As demonstrated by Baldwin et al. (1996), these methods can be applied successfully to optical interferometers as well. We present the first aperture synthesis images from IOTA3 in Figure 3, depicting $\lambda$ Vir for each epoch. The images are constructed iteratively starting with zero initial phases and using the measured closure phases for self-calibration. CLEAN deconvolution was applied using an elliptical Gaussian CLEAN beam. Full details of the image-making process for this source (as well as the binary Capella) can be found in Kraus (2003). The presented maps are consistent with the binary parameters found through direct fitting, and demonstrate the imaging capability of the IOTA3+IONIC-3 system, currently the most sensitive infrared imaging interferometer in the world.

With this new imaging capability, we begin to study binary stars which have never been resolved before and which represent unique stellar systems whose properties can shed new light on rare and/or short-lived phases of stellar evolution.

$\lambda$ Vir is a well-known spectroscopic binary (period 207 days, Stickland 1976) with similar components of spectral type early Am (both are metallic-lined, Abt 1961). The stars exhibit the curious property that one is sharp-lined and the other broad-lined, which makes them easily distinguishable spectroscopically. IOTA3 observations will allow a determination of the absolute properties of the components once the full orbit is established. Fundamental stellar parameters, such as the system distance (independent of Hipparcos) and the individual masses and absolute near-IR luminosities, can be determined through a combination of the radial velocity orbit, astrometric orbit, and brightness ratio. Further, these properties can be compared directly against models of stellar evolution to derive the age of the system. The very different projected rotational velocities of the components is of special interest for these Am stars, enabling unique tests of current theories of tidal evolution (Tassoul \& Tassoul 1997; Zahn \& Bouchet 1989, and references therein).

WR 140 is a prototypical colliding-wind binary source consisting of a Wolf-Rayet (WC7) and an O4-5 star in a highly-eccentric $\sim 7.9$ yr orbit, and has been extensively studied in the radio, infrared, optical, ultra-violet, and X-rays (e.g., Moffat et al. 1987; Williams et al. 1990; White \& Becker 1995). Notably, infrared emission appears and fades in conjunction with periastron passage, explained by transient dust formation in the dense shock-compressed gas at the wind-wind interface (Williams et al. 1990; Usov 1991; Tuthill et al. 1999). This process was captured in a series of high-resolution near-infrared images by Monnier et al. (2002), revealing an eastward moving arc of dust consistent with the O-star being roughly east of the WR star at periastron. The O-star is expected to be on (nearly) the opposite side of the WR star now as compared to periastron, which can be reconciled with the position angle 
found here $\left(\mathrm{PA} 152^{\circ}\right.$ ) only if the WR star is fainter than the O-star at $1.65 \mu \mathrm{m}$ (as expected by some workers; e.g. Williams et al. 1990). We intend to combine future high-precision interferometer observations with new radial velocity data (e.g., Marchenko et al. 2003) to derive component masses and a distance estimate, fundamental parameters for these systems needed for definitive interpretation of data across the electromagnetic spectrum.

\section{Conclusions}

We have reported first scientific results from the IOTA3 interferometer: newly-resolved separation, position angle, and flux ratio information for double-lined spectroscopic binary stars $\lambda$ Vir and WR 140. Using a preliminary data reduction pipeline, calibration is sufficient for model-fitting and aperture-synthesis imaging using hybrid mapping techniques. New observations are being pursued in order to derive precise component masses and distance estimates by combining interferometric and radial velocity data. All the visibility and closure

phase data here have been converted to the new FITS format for Optical Interferometry data (OI-FITS) ${ }^{1}$ and are available upon request.

The authors gratefully acknowledge critical support from SAO, NASA, and NSF (AST0138303). EP was supported by a SAO Predoctoral fellowship, JDM by a CfA fellowship, and RM-G, J-PB, and SR through NASA Michelson Fellowships. GT acknowledges partial support from NASA's MASSIF SIM Key project (JPL 1240033). In addition, we acknowledge useful contributions from B. Arezki, A. Delboulbe, C. Gil, S. Gluck, E. Laurent, R. B. Metcalf, and E. Tatulli. IONIC-3 was developed by LAOG and LETI in the context of the IONIC collaboration (LAOG, IMEP, LETI), funded by the CNRS and CNES (France). This publication makes use of data products from the 2MASS survey, which is a joint project of the University of Massachusetts and IPAC/Caltech, funded by NASA and the NSF. This research has also used the SIMBAD database, NASA's ADS Service, and services of the Michelson Science Center at Caltech (http://msc.caltech.edu).

\section{REFERENCES}

Abt, H. A. 1961, ApJS, 6, 37

Baldwin, J. E., et al., R. W. 1996, A\&A, 306, L13+

\footnotetext{
${ }^{1}$ http://www.mrao.cam.ac.uk/ jsy1001/exchange/
} 
Benson, J. A., et al. , C. S. 1997, AJ, 114, 1221

Berger, J., et al. 2003, in Interferometry for Optical Astronomy II. Edited by Wesley A. Traub . Proceedings of the SPIE, Volume 4838, pp. 1099-1106 (2003)., 1099-1106

Berger, J. P., et al. 2001, A\&A, 376, L31

Boden, A. F., et al. 1999, ApJ, 527, 360

Cornwell, T. J. \& Wilkinson, P. N. 1981, MNRAS, 196, 1067

Coudé du Foresto, V., Ridgway, S., \& Mariotti, J.-M. 1997, A\&AS, 121, 379

Haniff, C. A., Mackay, C. D., Titterington, D. J., Sivia, D., \& Baldwin, J. E. 1987, Nature, 328, 694

Hummel, C. A., Armstrong, J. T., Quirrenbach, A., Buscher, D. F., Mozurkewich, D., Elias, N. M., \& Wilson, R. E. 1994, AJ, 107, 1859

Hummel, C. A., Mozurkewich, D., Armstrong, J. T., Hajian, A. R., Elias, N. M., \& Hutter, D. J. 1998, AJ, 116, 2536

Jennison, R. C. 1958, MNRAS, 118, 276+

Kern, P., Malbet, F., Schanen-Duport, I., \& Benech, P. 1997, in Integrated Optics for Astronomical Interferometry (1996 Oct 15-16, Grenoble, France), 195-+

Kraus, S. 2003, Master's thesis, University of Massachusetts, Amherst

Marchenko, S. V., et al. 2003, ApJ, 596, 1295

Millan-Gabet, R., Schloerb, F. P., \& Traub, W. A. 2001, ApJ, 546, 358

Moffat, A. F. J., Lamontagne, R., Williams, P. M., Horn, J., \& Seggewiss, W. 1987, ApJ, 312, 807

Monnier, J. D. 2000, in Principles of Long Baseline Stellar Interferometry (JPL Publication 00-009), 203-+

Monnier, J. D. 2001, PASP, 113, 639

Monnier, J. D., Tuthill, P. G., \& Danchi, W. C. 2002, ApJ, 567, L137

Perrin, G. 2003, A\&A, 398, 385 
Readhead, A. C. S., Walker, R. C., Pearson, T. J., \& Cohen, M. H. 1980, Nature, 285, 137

Schloerb, F. P. 1990, in Amplitude and intensity spatial interferometry; (Tucson, AZ, Feb. 14-16, 1990) SPIE A91-30676 12-89, 154-165

Stickland, D. J. 1976, in IAU Colloq. 32: Physics of Ap Stars (eds. W. W. Weiss, H. Jenkner and H. J. Wood), 701-+

Tassoul, M. \& Tassoul, J. 1997, ApJ, 481, 363

Traub, W. A., et al. 2003, in Interferometry for Optical Astronomy II. Edited by Wesley A. Traub. Proceedings of the SPIE, Volume 4838, pp. 45-52 (2003)., 45-52

Tuthill, P. G., Monnier, J. D., \& Danchi, W. C. 1999, Nature, 398, 487

Usov, V. V. 1991, MNRAS, 252, 49

White, R. L. \& Becker, R. H. 1995, ApJ, 451, 352+

Williams, P. M., van der Hucht, K. A., Pollock, A. M. T., Florkowski, D. R., van der Woerd, H., \& Wamsteker, W. M. 1990, MNRAS, 243, 662

Zahn, J.-P. \& Bouchet, L. 1989, A\&A, 223, 112 
Table 1. Observing Log for $\lambda$ Vir and WR 140

\begin{tabular}{|c|c|c|}
\hline $\begin{array}{l}\text { Date } \\
\text { (UT) }\end{array}$ & $\begin{array}{l}\text { Interferometer } \\
\text { Configuration }^{(a)}\end{array}$ & $\begin{array}{c}\text { Calibrator Names } \\
\text { (Adopted UD Diameter) }\end{array}$ \\
\hline & \multicolumn{2}{|c|}{$\lambda \operatorname{Vir}(\mathrm{A} 1 \mathrm{~V}+\mathrm{A}) ; \mathrm{H}$ mag $=4.28 \pm 0.21$} \\
\hline 2003 Feb 16,17 & A35-B05-C10 & $\begin{array}{l}\text { HD } 126035 \text { (G7III, } 0.78 \pm 0.24 \text { mas) } \\
\text { HIP } 71957 \text { (F2III, } 1.20 \pm 0.22 \text { mas) }\end{array}$ \\
\hline 2003 Feb $20,22,23$ & A25-B15-C10 & HD 126035 \\
\hline 2003 Mar 21 & A35-B07-C25 & HD 126035 \\
\hline 2003 Mar 22 & A35-B07-C10 & HD 126035; HD $158352(\mathrm{~A} 8 \mathrm{~V}, 0.44 \pm 0.10)$ \\
\hline 2003 Mar 23-24 & A35-B15-C10 & HD 126035; HD 158352 \\
\hline 2003 Jun 12-17 & A35-B15-C10 & HD 126035 \\
\hline 2003 Jun 17 & $\begin{array}{l}\text { 140 (WC7 + O4 } \\
\mathrm{A} 35-\mathrm{B} 15-\mathrm{C} 10\end{array}$ & $\begin{array}{c}/ 5) ; \mathrm{H} \mathrm{mag}=5.429 \pm 0.023 \\
\text { HD } 192985 \text { (F5IV, } 0.46 \pm 0.10 \mathrm{mas}) \\
\text { HD } 193631 \text { (K0, } 0.44 \pm 0.10 \text { mas })\end{array}$ \\
\hline
\end{tabular}

Notes: H-band photometry from 2MASS All-Sky Release; calibrator diameters estimated using getCal software (http://msc.caltech.edu).

${ }^{a}$ Configuration refers to the location of telescopes $\mathrm{A}, \mathrm{B}, \mathrm{C}$ on the NE, SE and NE arm respectively; see $\S 2$ for more details. 
Table 2. Binary Parameters for $\lambda$ Vir and WR 140 at $1.65 \mu \mathrm{m}$

\begin{tabular}{llccccc}
\hline \hline Source & Epoch & Separation & Position Angle & Flux & \multicolumn{2}{c}{$\chi^{2} / \mathrm{DOF}^{(b)}$} \\
& (U.T.) & (milliarcseconds) & (degrees) & Ratio & $V^{2}$ & CP \\
\hline \multirow{2}{*}{$\lambda$ Vir } & 2003 Feb 16-23 & $18.2 \pm 0.6$ & $200 \pm 3$ & $1.69_{-0.21}^{+0.29}$ & 0.45 & 12.8 \\
& 2003 Mar 21-24 & $17.8 \pm 0.5$ & $184 \pm 3$ & $1.72 \pm 0.07$ & 0.33 & 2.2 \\
& 2003 Jun 12-17 & $19.5 \pm 0.6$ & $22 \pm 3$ & $2.05_{-0.35}^{+0.50}$ & 0.31 & 4.8 \\
\multirow{2}{*}{ WR 140 } & 2003 Jun 17 & $12.9_{-0.4}^{+0.5}$ & $151.7_{-1.3}^{+1.8}$ & $1.10_{-0.05}^{+0.06}$ & 0.13 & 4.7 \\
\hline
\end{tabular}

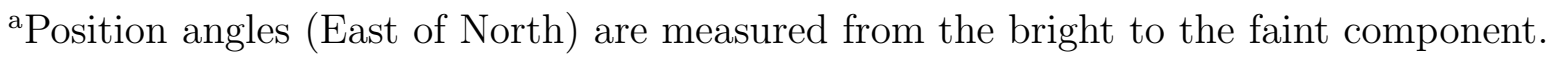

${ }^{\mathrm{b}}$ The $\chi^{2}$ per DOF (degree of freedom), the reduced $\chi^{2}$, is reported for the model fit to the $\mathcal{V}^{2}$ and closure phase $(\mathrm{CP})$ measurements separately. 


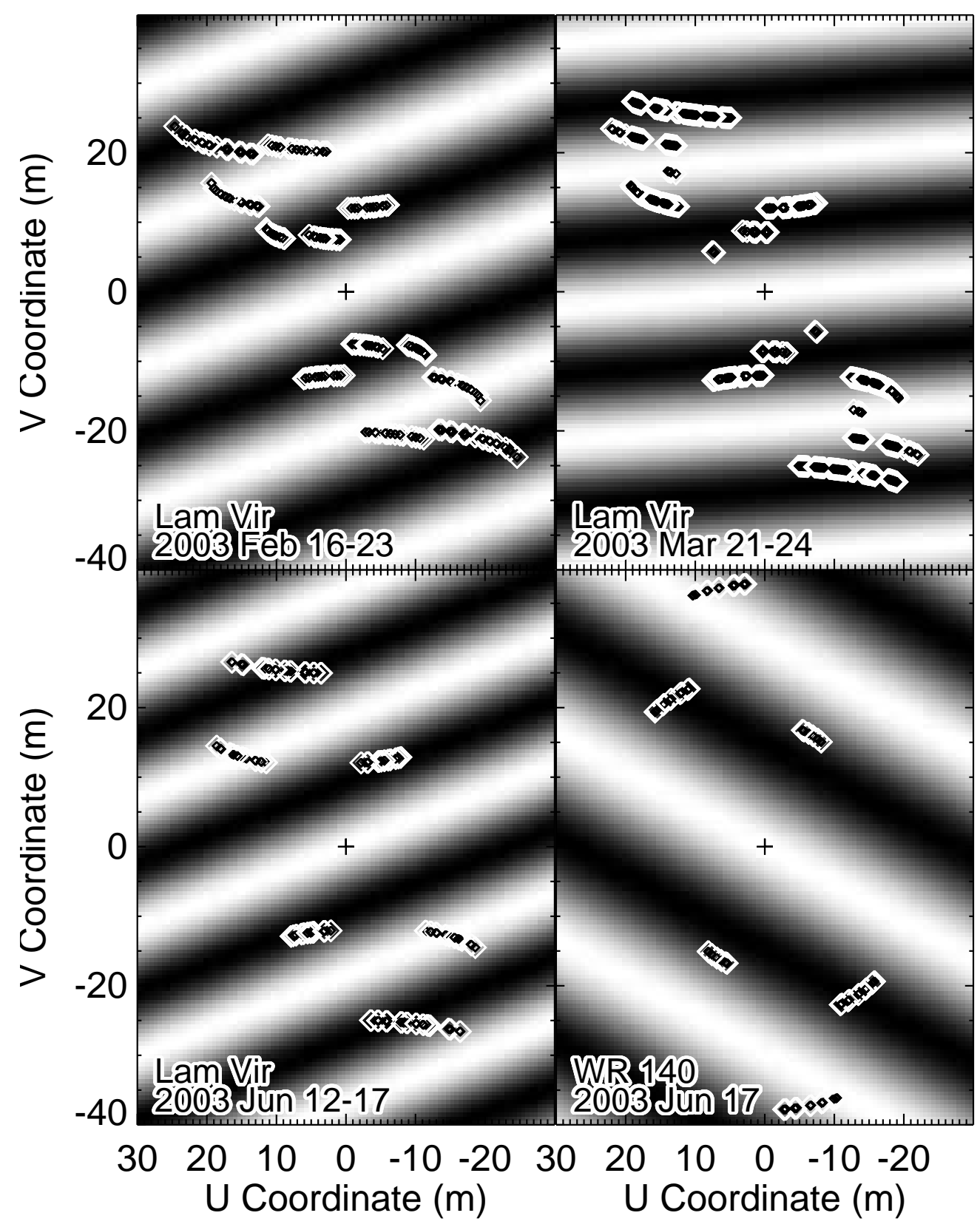

Fig. 1.- $(\mathrm{u}, \mathrm{v})$-plane coverage of science binaries. Each diamond symbol represents a single observation with the IOTA3 interferometer, corresponding to approximately 100-200 interferogram scans. The overlaid image in each panel represents the $\mathcal{V}^{2}$ of the best fit binary model for each epoch discussed in text. 

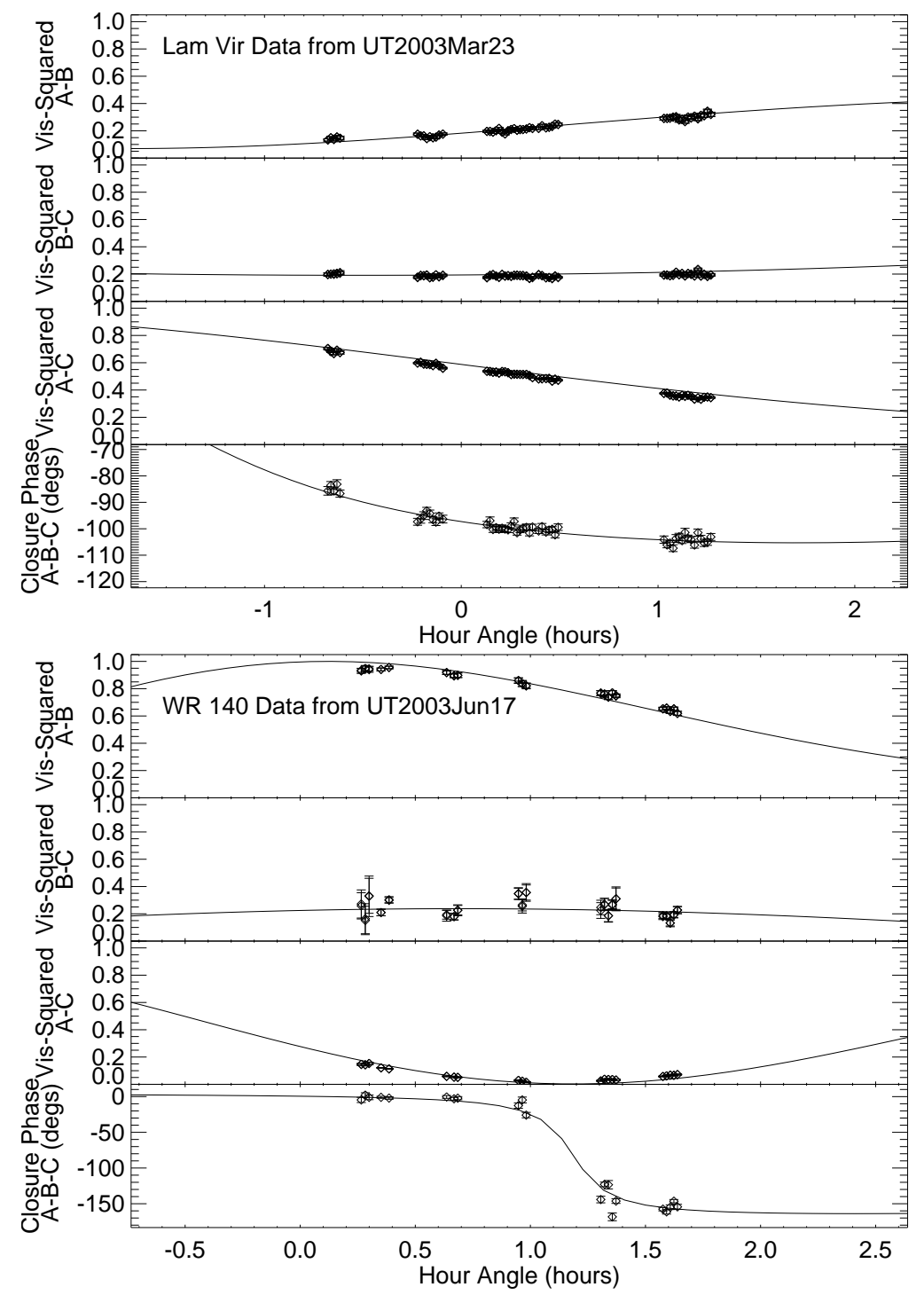

Fig. 2.- (top panel): This figure shows example binary model fits for $\lambda$ Vir on UT 2003 March 23. The top three panels show the $\mathcal{V}^{2}$ for baselines connecting telescopes $\mathrm{A}-\mathrm{B}, \mathrm{B}-\mathrm{C}$, and A-C, respectively. The bottom panel shows the calibrated closure phase for the A-B-C triangle. (bottom panel): Same as top panel for UT 2003 June 17 observations of WR 140. These curves represent the best-fit model parameters as described in $\S 3$. For all panels, the error bars shown represent only the statistical error and do not include the systematic error discussed in $\S 2$. 


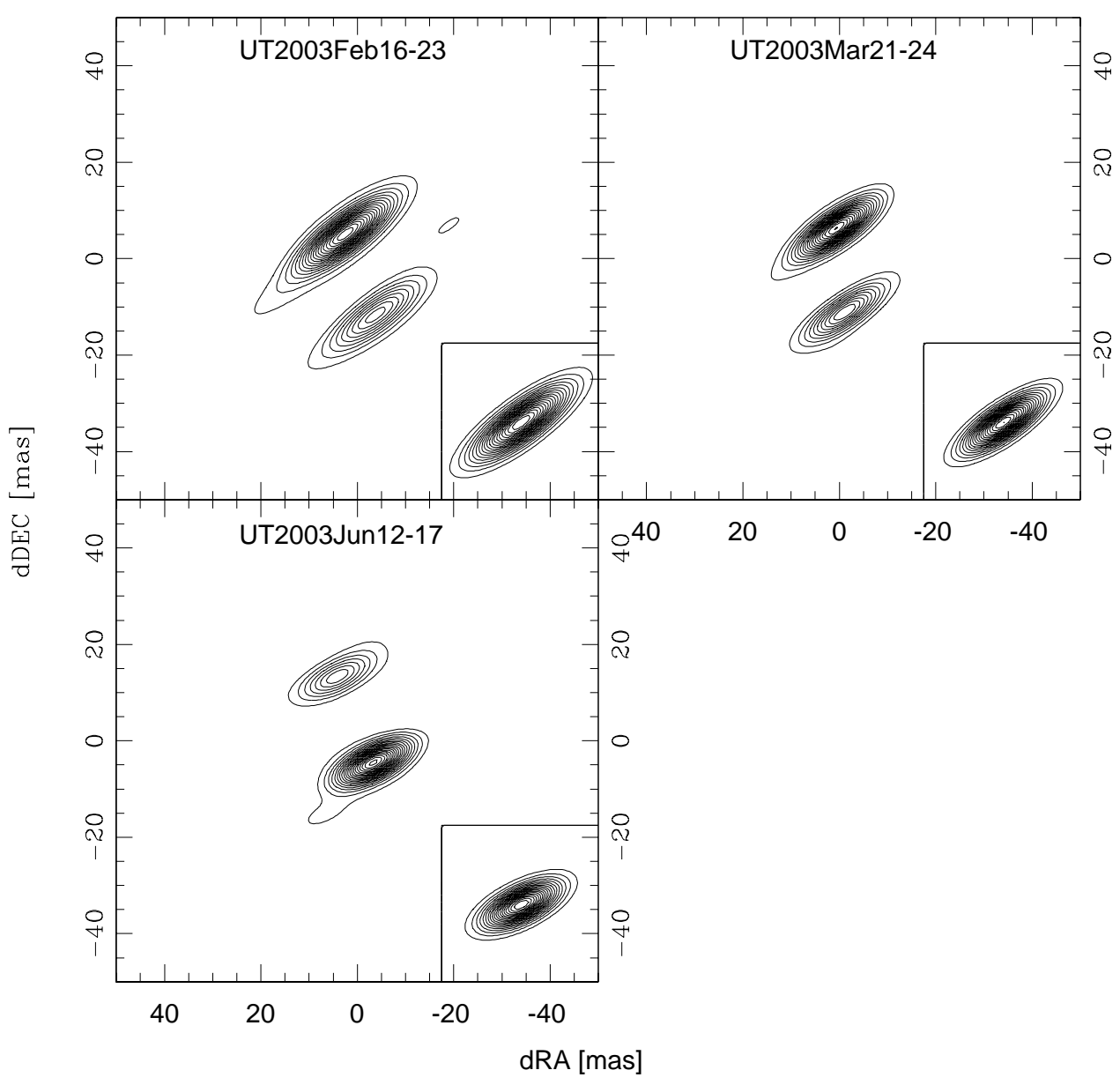

Fig. 3.- First aperture synthesis images from the upgraded IOTA 3-telescope interferometer, depicting $\lambda$ Vir based on $1.65 \mu \mathrm{m}$ observations at three separate epochs. The binary components are well-resolved from each other but individually are unresolved; orbital motion is clearly evident, showing nearly a $\sim 180^{\circ}$ rotation between the first and last epoch. Each panel includes a contour map showing $5 \%$ intervals in surface brightness scaled to the peak, as well as the the CLEAN restoring beam as inset. North is up and East is left. 